\title{
BMJ Global Health Preventing violence against refugee adolescent girls: findings from a cluster randomised controlled trial in Ethiopia
}

\author{
Lindsay Stark, ${ }^{1,2}$ Khudejha Asghar, ${ }^{1}$ Ilana Seff, ${ }^{1}$ Gary Yu,,${ }^{1,3}$ \\ Teame Tesfay Gessesse, ${ }^{4}$ Leora Ward, ${ }^{5}$ Asham Assazenew Baysa, ${ }^{5}$ Amy Neiman, ${ }^{5}$ \\ Kathryn L Falb ${ }^{5}$
}

To cite: Stark L, Asghar K, Seff I, et al. Preventing violence against refugee adolescent girls: findings from a cluster randomised controlled trial in Ethiopia. BMJ Glob Health 2018;3:e000825. doi:10.1136/ bmjgh-2018-000825

Handling editor Seye Abimbola

- Additional material is published online only. To view please visit the journal online (http://dx.doi.org/10.1136/ bmjgh-2018-000825).

Received 13 March 2018 Revised 30 July 2018 Accepted 3 August 2018

\section{SLinked}

- https://doi.org/10.1136/ bmjgh-2018-000824

Check for updates

(c) Author(s) (or their employer(s)) 2018. Re-use permitted under CC BY-NC. No commercial re-use. See rights and permissions. Published by BMJ.

For numbered affiliations see end of article.

Correspondence to

Dr Lindsay Stark;

Is2302@cumc.columbia.edu

\section{ABSTRACT}

Introduction Interpersonal violence is a critical public health concern in humanitarian contexts, but evidence of effective violence prevention programmes targeting adolescent girls is lacking. We investigated the efficacy of a life skills and safe spaces programme to reduce adolescent girls' experiences of interpersonal violence in a refugee setting.

Methods In this two-arm, single-blinded, cluster randomised controlled trial, we recruited 919 Sudanese and South Sudanese girls ages 13-19 years residing in refugee camps in Ethiopia. Girls were divided into 31 clusters, with 457 and 462 participants assigned to the intervention and control arms, respectively. Intervention clusters received 30 life skills sessions delivered in safe spaces and 8 complementary sessions for caregivers. The primary outcome was exposure to sexual violence in the previous 12 months. Secondary outcomes included disaggregated forms of sexual violence, physical violence, emotional violence, transactional sex, child marriage, feelings of safety, attitudes around rites of passage and perceptions of social support. Intent-to-treat analysis was used.

Results At 12-month follow-up, the intervention was not significantly associated with reduction in exposure to sexual violence (adjusted OR $=0.96,95 \% \mathrm{Cl} 0.59$ to 1.57 ), other forms of violence, transactional sex or feelings of safety. The intervention was associated with improvements in attitudes around rites of passage and identified social supports. Additionally, the intervention showed a decrease in reported child marriage among girls who were married at baseline.

Conclusion While the intervention impacted key markers along the causal pathway to violence reduction, further research and programmatic adaptations are needed to prevent violence towards adolescents in humanitarian contexts.

Trial registration NCT02506543.

\section{INTRODUCTION}

Throughout adolescence, individuals face unique and critical threats to health, well-being and security. ${ }^{1}$ Globally, adolescence marks the period of greatest risk of

\section{Key questions}

What is already known?

- While life skills programming has been identified as a promising intervention strategy to prevent gender-based violence in low-income and middle-income countries, it is currently unknown whether this strategy's potential extends to humanitarian settings.

\section{What are the new findings?}

- While we found no evidence that the Creating Opportunities through Mentorship, Parental Involvement, and Safe Spaces intervention reduced girls' exposure to sexual violence within the 12-month timeframe measured, findings indicate positive impact of the intervention on attitudes around rites of passage and social support indicators.

- Findings also showed a decrease in reports of child marriage among girls in the intervention who reported being married or living with a man at baseline.

What do the new findings imply?

- Given the complexities of running randomised controlled trials in humanitarian contexts, outcome measures may need to be tailored to align with realistic timelines and the realities of programming in emergency settings.

interpersonal violence victimisation for girls. ${ }^{23}$ Girls reaching reproductive age are vulnerable to multiple forms of violence, including child maltreatment, intimate partner violence (IPV), child marriage, sexual abuse and exploitation and female genital mutilation/cutting. ${ }^{4} 5$ Additionally, adolescent girls' low position in many societies may exacerbate risks of victimisation and impede service access and utilisation. These risks may be amplified by frequently occurring political and social upheaval during emergencies. ${ }^{67}$

Experiencing violence during childhood and adolescence can disrupt development trajectories, with greater impact than 
exposure at other periods in the life course ${ }^{8-10}$ Indeed, multiple forms of gender-based violence (GBV) experienced during childhood and adolescence are associated with long-term health consequences for females, including poor sexual and reproductive health, increased risk of HIV, physical injury and disability, poor mental well-being and suicidal ideation. ${ }^{11-15}$ Given that suicide and depressive disorders are the leading global causes of mortality and years lost to disability, respectively, for adolescent girls ages 15-19 years, reducing exposure to violence and promoting positive development may decrease the burden of mental ill-health and improve general health and well-being for adolescent girls. ${ }^{1617}$

Life skills and education interventions have demonstrated promising changes in adolescent girls' violence-related attitudes and behaviours in developing settings. ${ }^{18} 19$ These interventions have identified social norms related to gender and violence, adolescent girl empowerment and social networks, as pathways for reducing risk of GBV. Interventions have included safe spaces and combinations of life skills training, awareness-raising around girls' rights and violence and social network building. ${ }^{20}$ A few interventions have also included influential adults in girls' lives, such as caregivers, or facilitated access to a young female mentor. ${ }^{1921}$

To date, programming to prevent violence against adolescent girls in humanitarian settings has been limited. A recent systematic review of approaches to reducing GBV against adolescent girls in humanitarian settings found only three evaluations in the academic literature, all of which used weak pretest and post-test designs. ${ }^{22}$ Additionally, none of the 38 adolescent IPV prevention interventions identified in a recent Cochrane review were implemented in humanitarian settings. ${ }^{23}$ As suggested by these reviews, significant knowledge gaps persist around effective strategies for reducing experiences of violence and promoting positive development for adolescent girls in humanitarian settings.

To address this gap in evidence and practice, Columbia University and the International Rescue Committee (IRC) investigated the efficacy of the Creating Opportunities through Mentorship, Parental Involvement, and Safe Spaces (COMPASS) adolescent life skills and safe spaces programme for refugee adolescent girls. To our knowledge, this study contributes the first rigorous scientific learning of an intervention to reduce GBV and support empowerment of adolescent girls in a humanitarian setting. This study presents the results of a cluster randomised controlled trial to determine the impact of the COMPASS programme on experiencing interpersonal violence, social support and psychosocial outcomes for refugee adolescent girls living in Ethiopia.

\section{METHODS}

\section{Study design and participants}

COMPASS is a programme implemented with refugees living in camps on the Sudan/Ethiopia border, conflict-affected communities in eastern Democratic Republic of Congo (DRC) and displaced populations in northeast Pakistan. Columbia University evaluated COMPASS in all three locations, each of which had a different research objective and design. In Ethiopia, the impact evaluation studied whether the core programme components had an impact on girls' experiences of violence and social outcomes. The DRC study measured the incremental impact of a caregiver curriculum on girls' experiences of violence and social outcomes, along with attitudes and characteristics of participating caregivers. In Pakistan, the evaluation assessed the acceptability of the programme to adolescent girls and parents / caregivers in their context and measured changes in girls' social and health outcomes over the course of the programme. ${ }^{24}$ This article presents findings from the evaluation implemented in Ethiopia.

In this wait-list, cluster randomised controlled trial (see online supplementary file - CONSORT cluster trials checklist), adolescent girls ages 13-19 years were recruited from three refugee camps in the Benishangul-Gumuz regional state of Ethiopia. Ethiopia is a top asylum destination for refugees fleeing protracted conflicts in nearby countries, and Benishangul-Gumuz camps host tens of thousands of refugees from Sudan and South Sudan. Adolescent refugees from Sudan have been repeatedly found to exhibit elevated rates of depression, anxiety and post-traumatic stress. ${ }^{25}$ Additionally, a recent study from other Ethiopian refugee camps revealed that adolescent refugee girls are at risk of physical, emotional and sexual violence victimisation in this setting. ${ }^{26}$

Adolescents who met inclusion criteria were enrolled in the study from 29 July 2015 to 4 September 2016. Girls were excluded if they lacked verbal proficiency in Funj, Regarig, Engesena, or Maban. Language exclusion criteria were based on the primary languages spoken by girls in the research camps and data collectors' language abilities. Individuals with significant cognitive impairments or physical disabilities that would prevent independent completion of interviews using Audio Computer-Assisted Self-Interview (ACASI) were excluded for ethical reasons. All study procedures were approved by the Institutional Review Board of the Columbia University Medical Center (Protocol \#AAAP6855; see online supplementary file - IRB protocol), the Administration for Refugee and Returnee Affairs in Ethiopia and the IRC's internal review board (Protocol \# WPE 1.00.003).

\section{Study procedures}

IRC staff introduced the intervention to adolescent girls and their caregivers through home visits and existing programming at IRC's Women and Girls Wellness Centers. Girls who registered for the intervention were invited to participate in the study by data collectors who were not affiliated with the IRC programme and who were managed by Columbia University. Data collection trainers were all affiliated with the Care and Protection of Children Learning Network, and training 
included special attention to data collection with young people derived from a series of guidance materials. ${ }^{27-29}$ All research staff participated in a training focused on ensuring privacy and confidentiality and understanding the basic elements of GBV as outlined in the WHO's guidelines on violence against women research. Data collectors signed confidentiality agreements and developed protocols for data breaches. ${ }^{30}$ In accordance with ethical protocols for research in non-written languages, data collectors obtained verbal consent in confidential spaces from guardians, adolescent girls over age 18 years, and married girls below age 18 years, and assent for unmarried girls age 13-17 years. Adolescents self-administered the survey, using ACASI programming in a private location. ${ }^{30}$ Data collectors remained outside confidential spaces to respond to questions and to troubleshoot any technological difficulties. Following the interview, participants were provided with information on how to access violence and psychosocial support services, and referrals were made to service providers as needed.

Given that girls received the COMPASS intervention in small programme groups, we used these groups to cluster treatment assignments. Following completion of baseline data collection, adolescents were divided into 62 programme clusters of 10-20 girls based on household proximity, age (13-14 years or 15-19 years) and primary language. Columbia University randomised clusters to the treatment or wait-list control arm using a uniform distribution in R. To reduce contamination potential, siblings were randomised to the same treatment arm. Given the nature of programming received in the intervention arm, randomisation was blinded to data collectors but not blinded for participants.

Participants in both treatment arms were tracked and retained through strategies such as brief home visits occurring 6 months after baseline data collection and a visit 1 month prior to endline survey administration. To ensure the girls completing the endline surveys were the same as those from baseline, identifying information was first checked against a master list from baseline. Girls were then asked a series of questions about their baseline interview, such as what materials were used and where interviews were held. Adolescents completed the endline survey approximately 1 year after completion of the baseline survey (July-September 2016). Nested qualitative data were also collected and are presented elsewhere. ${ }^{32} 33$ All data collection took place in empty classrooms in local schools.

\section{COMPASS intervention}

Previous IRC learning on programming for children and adolescent girls informed intervention development. Intervention components included weekly adolescent girl life skills sessions in safe spaces, with 45-60 min of facilitated content and $30 \mathrm{~min}$ of unstructured time. Adolescents in clusters randomised to the treatment arm received a total of 30 in-person group sessions. Each girl's life skills session focused on topics related to improving key skills such as communication, friendship building, and awareness of GBV and sexual and reproductive health (see online supplementary appendix A. Girls' sessions were delivered by trained female refugee mentors aged 18-30 years, who spoke the same languages as participants. Groups were led by either one mentor or a team of two or three mentors.

The intervention also included 8 monthly discussion groups for enrolled girls' caregivers, which covered topics such as communication skills, supporting adolescent girls and understanding violence and abuse. Caregiver content was delivered by IRC staff with assistance from translators. Safe spaces were accessible to all women and girls living in the camps for unstructured activities in between COMPASS sessions. ${ }^{30}$

The COMPASS theory of change hypothesised that weekly discussion sessions for adolescent girls, access to a trusted female mentor and provision of safe singlegender space would increase girls' human, social, physical and financial assets to protect themselves from a range of potential risks, including IPV, community violence and transactional sex, as well as respond to threats or incidents of such violence (see figure 1). Building on previous experience with the Parents Make a Difference programme with younger children, it was also theorised that including caregivers could increase their protective role in girls' lives both within and outside the home, by raising their awareness about the risks of GBV faced by adolescent girls in their care and the importance of connecting girls with GBV response and health services. ${ }^{28}$

\section{Measures}

\section{Primary outcomes}

The primary outcome of interest was a binary composite representing any form of sexual violence in the previous 12 months, which included self-reported forced sex, unwanted sexual touching or coerced sex.

\section{Secondary outcomes}

Secondary outcomes included binary self-reported 12-month exposure to forced sex (having sex unwillingly), unwanted sexual touching and coerced sex in the previous 12 months. These measures are the disaggregated components of the primary outcome and were adapted from previously validated questionnaires. ${ }^{34}$ Other secondary outcomes included binary self-reported 12-month exposure to physical violence, emotional violence, transactional sex, child marriage and feelings of safety. Physical violence was operationalised as being hit or beaten. Emotional violence was defined as being screamed at loudly or aggressively. Engagement in transactional sex was operationalised as exchanging sex for money, food or gifts. Child marriage was defined as marrying or living with someone as if married prior to 18 years of age. Adolescents similarly provided binary responses on their feelings of safety in their homes, schools, friend's homes and neighbour's homes. Each of these variables on self-reported safety was operationalised 
PROBLEM

\begin{tabular}{|c|c|c|c|c|}
\hline \multicolumn{5}{|c|}{$\begin{array}{l}\text { Violence against adolescent girls in humanitarian settings in Ethiopia, Pakistan and the Democratic Republic of Congo inflicts long- } \\
\text { lasting physical and emotional harm, violates their rights, and impedes their ability to pursue safe, healthy and productive lives. }\end{array}$} \\
\hline RISKS & INTERVENTIONS & OUTPUTS & OUTCOME & IMPACT \\
\hline $\begin{array}{l}\text { Lack of safe and } \\
\text { empowering } \\
\text { opportunities for } \\
\text { adolescent girls to } \\
\text { increase their } \\
\text { resilience to violence }\end{array}$ & $\begin{array}{l}\text { Provide opportunities for } \\
\text { girls to build assets to } \\
\text { protect against and } \\
\text { respond to violence } \\
\text { through mentorship, } \\
\text { learning and peer } \\
\text { interaction in safe spaces }\end{array}$ & $\begin{array}{l}\text { Girls have increased } \\
\text { human, social, physical } \\
\text { and financial assets to } \\
\text { protect themselves from } \\
\text { violence and respond to } \\
\text { threats or incidents of } \\
\text { violence }\end{array}$ & \multirow{4}{*}{$\begin{array}{c}\text { Improved } \\
\text { prevention of } \\
\text { and response to } \\
\text { violence against } \\
\text { adolescent girls } \\
\text { in humanitarian } \\
\text { settings, } \\
\text { particularly in } \\
\text { Ethiopia, } \\
\text { Pakistan and } \\
\text { DRC }\end{array}$} & \multirow{4}{*}{$\begin{array}{l}\text { Adolescent girls } \\
\text { in humanitarian } \\
\text { settings are } \\
\text { safer from } \\
\text { violence and } \\
\text { the threat of } \\
\text { violence }\end{array}$} \\
\hline $\begin{array}{l}\text { Negative attitudes } \\
\text { and lack of capacity } \\
\text { of service providers } \\
\text { to meet the needs of } \\
\text { adolescent girls at } \\
\text { risk of violence }\end{array}$ & $\begin{array}{l}\text { Implement capacity } \\
\text { building activities to } \\
\text { improve the attitudes, } \\
\text { skills and practices of } \\
\text { service providers }\end{array}$ & $\begin{array}{l}\text { Existing service providers } \\
\text { (e.g. health, education, } \\
\text { case management) have } \\
\text { increased capacity to } \\
\text { provide safe, girl-friendly } \\
\text { and life-saving services }\end{array}$ & & \\
\hline $\begin{array}{l}\text { Negative beliefs, } \\
\text { attitudes and } \\
\text { behaviours among } \\
\text { girls' family } \\
\text { members, partners, } \\
\text { etc. that increase } \\
\text { risk of violence }\end{array}$ & $\begin{array}{l}\text { Conduct targeted } \\
\text { engagement (e.g. } \\
\text { discussion sessions) with } \\
\text { girls' family members, } \\
\text { partners, etc. to change } \\
\text { negative beliefs, attitudes } \\
\text { and behaviours }\end{array}$ & $\begin{array}{l}\text { Influential people in girls' } \\
\text { lives have improved } \\
\text { attitudes, knowledge and } \\
\text { skills to protect girls from } \\
\text { violence and support girls } \\
\text { to be safe from violence }\end{array}$ & & \\
\hline $\begin{array}{l}\text { Weak knowledge } \\
\text { base on prevention } \\
\text { of and response to } \\
\text { violence against } \\
\text { adolescent girls in } \\
\text { humanitarian } \\
\text { settings }\end{array}$ & $\begin{array}{l}\text { Conduct rigorous and } \\
\text { participatory research, } \\
\text { monitoring and evaluation } \\
\text { to produce applicable and } \\
\text { transferable learning for } \\
\text { the broader humanitarian } \\
\text { community }\end{array}$ & $\begin{array}{l}\text { Humanitarian community } \\
\text { has improved knowledge } \\
\text { of the risks of violence } \\
\text { faced by adolescent girls } \\
\text { in humanitarian settings } \\
\text { and how to respond } \\
\text { effectively to those risks }\end{array}$ & & \\
\hline
\end{tabular}

Figure 1 COMPASS Theory of Change. DRC, Democratic Republic of Congo.

as answering 'yes' to questions such as 'Do you feel safe at home?' and 'Do you feel safe at school?' and in a range of other settings where the IRC thought feelings of safety might be increased as a result of programming.

\section{Key causal pathway markers}

Key markers thought to influence girls' risk of experiencing interpersonal violence included social support and attitudes regarding rites of passage. Perceived social support was captured through dichotomous self-report of having female friends of a similar age outside the family and reports of having a trusted non-family female adult in whom they could confide. Rites of passage included adolescents' beliefs about the highest grade girls should complete in school, acceptability for girls to work outside the home after marriage, appropriate age of marriage and appropriate age of having one's first child. Continuous measures for appropriate age of marriage and age of first child were dichotomised as either under age 18 years or age 18 years and above. Adolescents also self-reported access to a safe place to spend time with other girls.

\section{Demographic variables}

Age and years of completed education were continuous variables. Cohabitation with biological parents was operationalised as a categorical variable: presence of only the father, only the mother, both parents, or neither parent in the home. Cohabitation with an intimate partner was defined as living with a husband or living with someone as if married.

\section{Statistical analysis}

The target sample size was originally calculated assuming 20 girls in each cluster; however, programme realities in the field led to fewer girls being assigned to each group and thus necessitated a recalculation of our sample size. We assumed $30 \%$ incidence of past-year sexual violence among the population at baseline and that this incidence was likely to remain constant among the control group. To calculate the target sample size, we assumed statistical power of $80 \%$ and a two-sided alpha of 0.05 to detect a $35 \%$ reduction in the incidence of past-year sexual violence in the intervention arm compared with the waitlist arm. We estimated that each cluster would comprise approximately 15 girls. We assumed an intraclass correlation coefficient (ICC) of 0.06 to account for clustering. We could not find a previous study that measured sexual violence among female adolescents in a sub-Saharan African site. However, a study that assessed IPV perpetrated against females ages 15-49 years across multiple sites, including Ethiopia, reported all ICCs were less than $0.06 .{ }^{35}$ We required 62 clusters, 31 groups in each treatment arm. We expected a $10 \%$ loss to follow-up, necessitating a final sample size of at least 896 girls.

Ranges, frequencies, means and SD were assessed for all predictors and outcomes (see online supplementary 
file - statistical analysis plan). t-Tests and Pearson $\chi^{2}$ tests were used to examine the independence of continuous and dichotomous baseline characteristics, respectively, between treatment arms and between those who were and were not lost to follow-up. Intent-to-treat analysis was conducted on all individuals surveyed at baseline. Per-protocol analysis is not presented due to the fact that more than $95 \%$ of girls and $91 \%$ of caregivers followed protocol, defined as attending at least $75 \%$ of programme sessions. The intervention's effects on binary primary and secondary outcomes at endline were assessed through mixed effects logistic regressions with random intercepts to account for clustering; the intervention's effect on a continuous secondary outcome was assessed using a linear mixed model. After estimating the first-order effect of the intervention on these outcomes, models were adjusted for baseline age, previous engagement in a romantic relationship and presence of mother, father or both parents in the home. These demographic characteristics were previously found to be associated with experiences of violence in baseline results of this study sample. ${ }^{36}$

Given attrition between baseline and follow-up and the self-reported and sensitive nature of many of the questions, outcomes were missing for approximately $10 \%-20 \%$ of girls. Pearson $\chi^{2}$ tests were used to assess whether missingness was associated with treatment assignment. We also carried out a sensitivity analysis by performing the analysis described above on imputed data. To account for the missing predictors and outcomes, we used a multiple imputation approach. A set of five imputations was generated in Stata with 'mi impute', after which the average values represented a close estimate of a full dataset. All analyses were completed using Stata V.14.

\section{RESULTS}

Of the 986 adolescent girls approached between 29 July 2015 and 4 September 2015, 919 girls completed the baseline survey (see figure 2). Adolescents were divided into clusters based on age, common language and geographic location, with an average of 15 girls per cluster. Thirty-one clusters were randomised to the intervention $(n=457)$ and 31 clusters were randomised to the waitlist arm $(n=462)$. Of these, $812(88 \cdot 36 \%)$ participants completed the endline survey between 25 July 2016 and 2 September 2016, and all 62 clusters were included in the analysis. The most frequently cited reason for non-completion of an endline survey was relocation out of the camps $(n=37)$. Additionally, 34 girls from the control group mistakenly joined the intervention and, due to an error in the field, endline data were not collected for these girls.

The average participant was approximately 14.5 years and had 2 years of schooling. At baseline, adolescents in the intervention group were more likely to live with their mother but not father and less likely to live with neither parent at baseline, as compared with the control group (table 1). Information on perpetrators of violence at baseline has been reported separately. ${ }^{36}$ The proportion

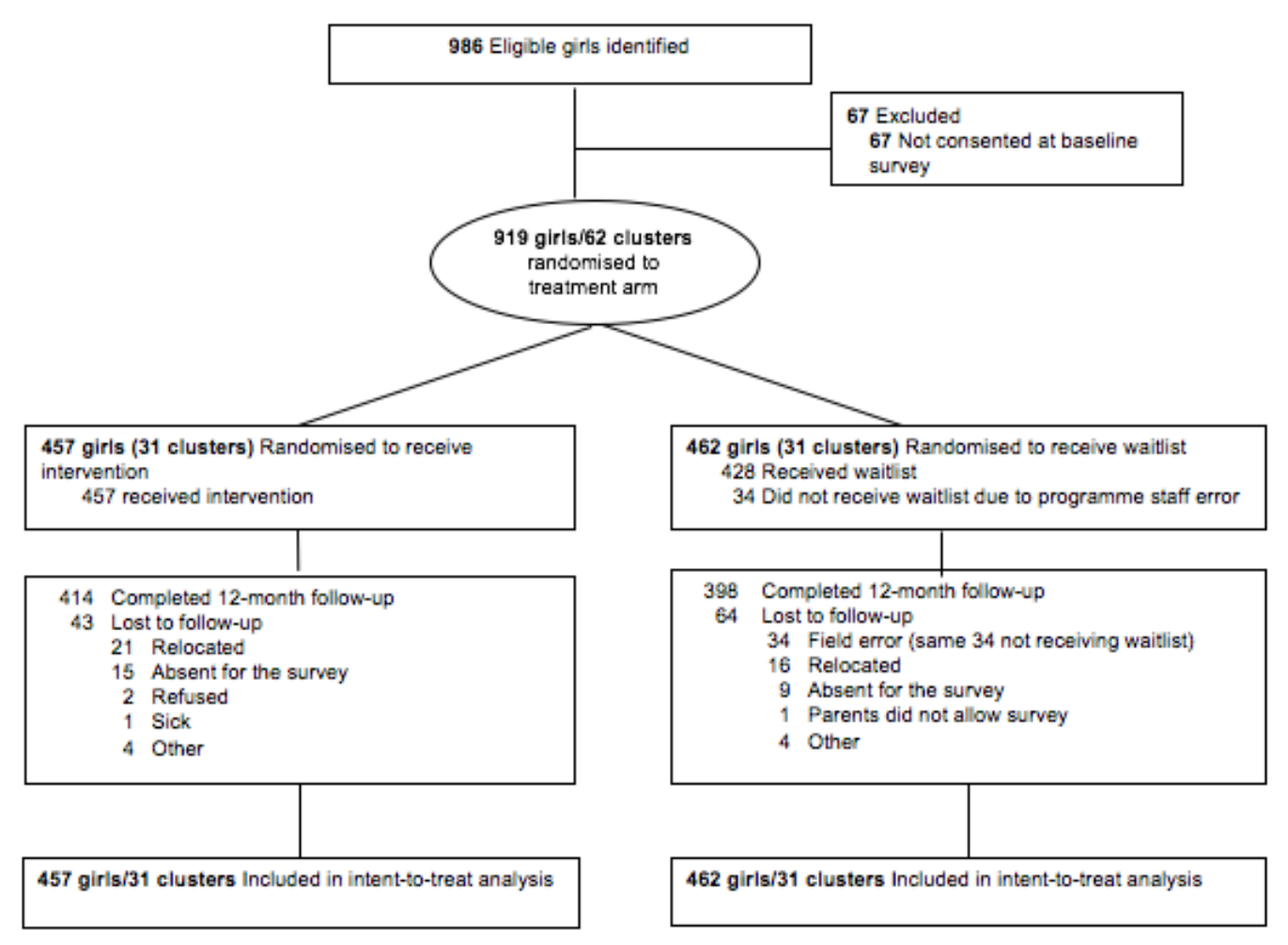

Figure 2 Trial Profile. 
Table 1 Baseline characteristics of girls in the treatment and waitlist arms

\begin{tabular}{|c|c|c|c|}
\hline Characteristics & Waitlist $(n=462)$ & Treatment $(n=457)$ & Missing \\
\hline \multicolumn{4}{|l|}{ Demographics } \\
\hline Age & $14.56(1.50)$ & $14.65(1.51)$ & 0 \\
\hline Language & & & 0 \\
\hline Engesena & $41(9)$ & $52(11)$ & \\
\hline Funj & $282(61)$ & $327(72)$ & \\
\hline Maban & $86(19)$ & $68(15)$ & \\
\hline Regarig & $53(11)$ & $10(2)$ & \\
\hline Camp & & & 0 \\
\hline Bambasi & $143(31)$ & $193(42)$ & \\
\hline Tongo & $166(36)$ & $117(26)$ & \\
\hline Sherkole & $153(33)$ & $147(32)$ & \\
\hline Parents in household & & & 39 \\
\hline Father only & $94(21)$ & $79(18)$ & \\
\hline Mother only & $105(24)$ & $135(31)$ & \\
\hline Both parents & $199(45)$ & $196(45)$ & \\
\hline Neither parent & $46(10)$ & $26(6)$ & \\
\hline Ever had a boyfriend & $139(26)$ & $142(34)$ & 81 \\
\hline Relationship status & & & 93 \\
\hline Single & $269(64)$ & $258(64)$ & \\
\hline Married, living with husband & $76(18)$ & $73(18)$ & \\
\hline Married, not living with husband & $44(10)$ & $53(13)$ & \\
\hline Living with man as if married & $34(8)$ & $19(5)$ & \\
\hline Years of schooling & $2.00(2.04)$ & $2.00(2.09)$ & 41 \\
\hline
\end{tabular}

Data are mean [SD] or number (\%). Some percentages do not add up to 100 because of rounding.

of adolescents speaking the four different languages also differed between the intervention and control groups, which was an expected effect of randomising siblings to the same treatment condition. Adolescents in the intervention and control groups did not differ on any other measured demographic characteristics or primary or secondary violence outcomes.

Baseline data revealed high levels of exposure to any form of past-year sexual violence: $28 \%$ of girls in the intervention arm and $30 \%$ of girls in the control arm reported experiencing any form of sexual violence in the past year. Table 2 summarises primary and secondary outcomes by treatment arm and time period of data collection and treatment. There were no differences in primary or secondary outcomes between the treatment and wait-list control groups at baseline. Additionally, girls were no more or less likely to have missing outcome data at endline based on treatment arm.

Due to unforeseen challenges associated with programme adaptation and implementation, the COMPASS intervention roll-out was delayed, and the reference period for the primary outcome thus extends to the beginning of the programme. As such, the secondary outcomes presented may be more relevant and appropriate, given the condensed timeline. While a third round of data collection was sought to assess longer term changes in violence reduction, this additional round of data collection was ultimately not possible due to funding limitations.

Analyses showed no significant effect of the intervention on reports of sexual violence (adjusted OR $(\mathrm{aOR})=0.96,95 \%$ CI 0.59 to 1.57$)$ in the previous 12 months, when adjusting for other covariates (see table 3 ). The ICC for sexual violence in our sample was 0.009. Analyses also showed no significant effect of the intervention on reports of specific forms of sexual violence, physical violence, emotional violence or transactional sex in the previous 12 months, as well as no effect on perceived feelings of safety (see table 3 ). While there was no difference in child marriage across treatment arms at endline, exploratory analyses revealed the intervention had an effect on child marriage for the subgroup of girls already married at baseline; among those married or living with someone as if married at baseline, girls in the treatment arm had lower odds (OR $0.57 ; 95 \%$ CI 0.34 to $0.95), \mathrm{p}=0.032$ ) of being married at endline as compared with those in the control arm (results not presented in table 3).

Adolescents in the intervention reported that they believed girls should complete one additional year of 
Table 2 Descriptive statistics for outcomes by time period and treatment arm

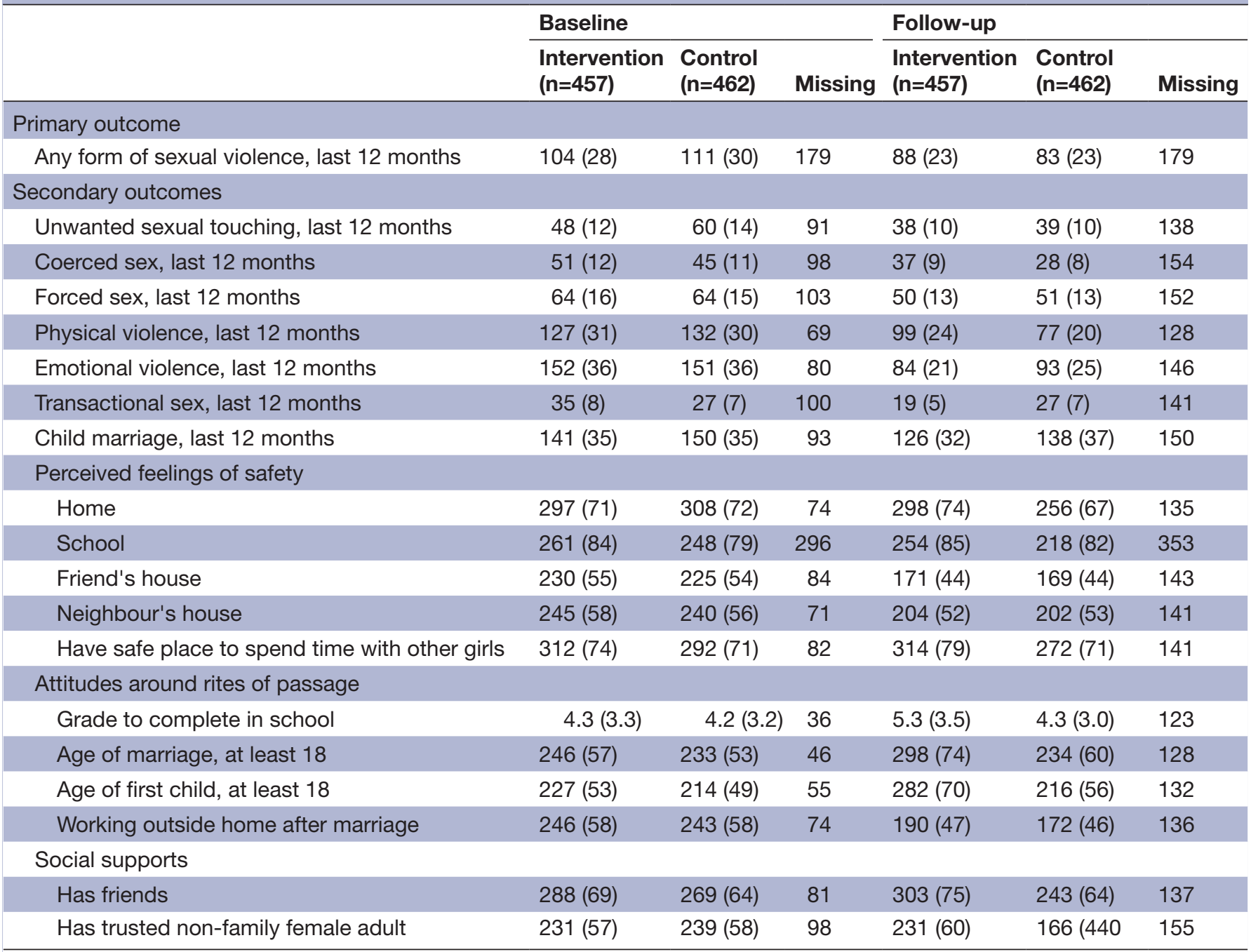

Data are mean [SD] or number (\%). Some percentages do not add up to 100 because of rounding.

schooling as compared with those in the control group $(\beta=1.08,95 \%$ CI 0.44 to 1.761$), p=0.001)$. Girls in the treatment arm also had greater odds than girls in the control arm of believing a girl should get married $(\mathrm{aOR}=1.88,95 \%$ CI 1.07 to $3.28, \mathrm{p}=0.027)$, and have her first child after age $18(\mathrm{aOR}=2.04,95 \%$ CI 1.25 to $3.34, p=0.005)$. Finally, girls in the intervention had 1.71 greater odds (95\% CI (1.18 to $2.49, \mathrm{p}=0.005)$ of reporting having friends their own age and 1.997 greater odds $(95 \%$ CI 1.44 to $2.76, \mathrm{p}<0.001$ ) of having a trusted non-family female adult in their life, when adjusting for other covariates. All findings are robust to sensitivity analyses (see online supplementary appendix B).

\section{DISCUSSION}

The COMPASS intervention's theory of change, built on learnings from The Parents Make a Difference programme and other studies, hypothesised that increasing girls' knowledge of violence risk factors, reshaping attitudes about gender roles, expanding social networks and improving decision-making and negotiation skills would ultimately equip girls to protect themselves against and respond to violence. ${ }^{30}$ While self-reported exposure to the primary outcome of sexual violence did decrease in both the control and intervention groups, we find no evidence that this decline was due to the intervention. However, although the programme did not impact girls' likelihood of experiencing sexual, physical or emotional violence, or transactional sex within the timeframe measured, our results suggest that the intervention did affect changes in gender attitudes and social support networks, which lie earlier along the causal pathway.

These results may reflect a need for a longer period of follow-up to document changes in violence, or may reflect a limited ability of the intervention to impact violence outcomes. It may be unreasonable to expect these standalone interventions to impact outcomes at every stage of the causal pathway, especially in a 12-month period, when there are many other structural factors that influence a girl's risk of experiencing violence. Leveraging opportunities for policy change through work with local leaders and other implementing organisations may expand the 
Table 3 Estimate of intervention effects

\begin{tabular}{|c|c|c|c|c|c|c|}
\hline & OR & $95 \% \mathrm{Cl}$ & Observations & aOR & $95 \% \mathrm{Cl}$ & Observations \\
\hline \multicolumn{7}{|l|}{ Primary outcome } \\
\hline Any form of sexual violence & 1.04 & 0.65 to 1.66 & 740 & 0.96 & 0.59 to 1.57 & 663 \\
\hline \multicolumn{7}{|l|}{ Secondary outcomes } \\
\hline Unwanted sexual touching & 0.93 & 0.52 to 1.65 & 781 & 0.86 & 0.48 to 1.54 & 695 \\
\hline Coerced sex & 1.31 & 0.75 to 2.29 & 765 & 1.20 & 0.66 to 2.19 & 680 \\
\hline Forced sex & 1.00 & 0.55 to 1.79 & 767 & 1.03 & 0.58 to 1.84 & 681 \\
\hline Physical violence & 1.30 & 0.81 to 2.09 & 791 & 1.17 & 0.73 to 1.89 & 699 \\
\hline Emotional violence & 0.82 & 0.58 to 1.16 & 773 & 0.88 & 0.60 to 1.29 & 688 \\
\hline Transactional sex & 0.65 & 0.35 to 1.23 & 778 & 0.66 & 0.33 to 1.32 & 690 \\
\hline Child marriage & 0.79 & 0.50 to 1.24 & 769 & 0.72 & 0.46 to 1.15 & 683 \\
\hline \multicolumn{7}{|l|}{ Perceived feelings of safety } \\
\hline Home & 1.55 & 0.91 to 2.62 & 784 & 1.57 & 0.96 to 2.57 & 694 \\
\hline School & 1.24 & 0.71 to 2.18 & 566 & 1.37 & 0.75 to 2.49 & 507 \\
\hline Friend's house & 1.09 & 0.58 to 2.05 & 776 & 1.06 & 0.57 to 2.00 & 690 \\
\hline Neighbour's house & 0.99 & 0.60 to 1.64 & 778 & 1.04 & 0.62 to 1.73 & 688 \\
\hline Safe place to spend time with other girls & 1.57 & 0.96 to 2.56 & 778 & 1.52 & 0.97 to 2.39 & 688 \\
\hline \multicolumn{7}{|l|}{ Attitudes around rites of passage } \\
\hline Grade to complete in school $(\beta(95 \% \mathrm{Cl}))$ & $0.93^{* *}$ & 0.31 to 1.56 & 796 & $1.08^{\star *}$ & 0.44 to 1.761 & 702 \\
\hline Age of marriage & $2.00^{*}$ & 1.16 to 3.46 & 791 & $1.88^{*}$ & 1.07 to 3.28 & 699 \\
\hline Age of first child & $1.94^{*}$ & 1.16 to 3.27 & 787 & $2.04^{\star \star}$ & 1.25 to 3.34 & 694 \\
\hline Working outside the home after marriage & 1.08 & 0.74 to 1.56 & 783 & 1.18 & 0.82 to 1.69 & 692 \\
\hline \multicolumn{7}{|l|}{ Social supports } \\
\hline Have friends & $1.83^{* *}$ & $1.20,2$ to 78 & 782 & $1.71^{\text {** }}$ & 1.18 to2.49 & 694 \\
\hline Have trusted non-family female adult & $1.89^{\star \star *}$ & 1.38 to 2.57 & 764 & $2.00^{\star \star \star}$ & 1.44 to 2.76 & 681 \\
\hline
\end{tabular}

No allowance for multiplicity was made in the analyses. Adjusted models control for having ever had a boyfriend, a four-level categorical variable indicating biological parents living in the home and age. All results are ORs or aORs, except for 'grade to complete in school'. ORs are statistically significant.at ${ }^{*} \mathrm{P}<0.05$, ${ }^{*} \mathrm{P}<0.01,{ }^{* * *} \mathrm{P}<0.001$.

aOR, adjusted OR.

intervention's effectiveness. ${ }^{29}$ Since caregivers and girls' intimate partners are frequent perpetrators of violence, including gender-transformative programming that targets these two groups may also improve reach of the intervention. Indeed, research indicates positive effects of community-level advocacy and social norms interventions on preventing IPV among women and parenting interventions targeting fathers on violence against children; similar programme adaptations and expansions for adolescent girls may strengthen positive outcomes of life skills programming like COMPASS. ${ }^{37-39}$ Furthermore, given that the inclusion of activities to target broader social norms in the community may promote positive and sustainable outcomes for girls and families, future evaluations of such programme should consider including measures of change at the community level, rather than just at the level of the girl participants.

Additionally, future programme might benefit from targeting social norms and behaviours among men in the community. Qualitative research activities conducted at baseline, including a participatory mapping exercise in which female adolescents were asked to draw their communities and identify 'safe' and 'unsafe' spaces, revealed the perceived threat of spaces that are dominated by male presence. ${ }^{32}$ Furthermore, girls agreed that most places are not inherently dangerous, but rather they pose a threat to girls' safety when occupied by men. However, this activity masked the incidence of caregiver and domestic violence in the private sphere as revealed through quantitative data collection, speaking to the importance of mixed methods in understanding the complexities of girls' experiences in these settings. ${ }^{40}$ Similar qualitative research should be conducted ahead of future programme in order to elucidate a topology of unsafe spaces as well as girls' perceived and real risks. Qualitative scenarios delivered through in-depth interviews are also recommended. ${ }^{33}$

Additional exploratory analyses of these data showed that the intervention was associated with a reduction in child marriage among girls who were married or living with someone as if married prior to age 18 years at baseline. These findings may indicate actual changes in adolescent girls' marital status due to increased awareness about the harms of child marriage or that social desirability bias made girls less likely to report being married at endline. 
Anecdotal validation from IRC staff confirmed that many girls who were living with partners as if married left these relationships over the course of the intervention period, underscoring that programme like COMPASS may offer greater benefits to some girls than others and such subgroup analyses are important in recognition that adolescent girls' experiences in humanitarian settings are not uniform.

A previous Lancet review of interventions to reduce violence against women and girls found insufficient evidence to advocate for empowerment interventions similar to COMPASS in high-income countries; however, the review found these strategies to be promising in low-income and middle-income countries. ${ }^{31}$ Our findings suggest that additional investigation is needed to explore the evidence for these strategies in humanitarian settings. Further research into the moderating and mediating role of prior exposure to outcome variables at baseline may illuminate mixed results on programme impact for girls with different vulnerabilities. Additionally, future analyses may consider factors associated with positive outcomes, such as improvements in social support, to discern associations between prior exposure to violence and feelings of social support after programme participation. Finally, previous research posits that a range of safety and coping strategies may support girls' adaptive resilience in settings where violence remains a salient threat. ${ }^{30}$ More realistic targets, given the theory of change, might have included reducing the frequency of violence victimisation or addressing girls' resilience in the face of violence, rather than primary prevention outright.

Despite the lack of evidence supporting the intervention's impact on violence outcome for girls, the programme's effects on other aspects of well-being and the high rates of programme adherence demonstrate the potential for implementing similar programme in humanitarian settings. Although there were few competing activities providing support for adolescent girls in the refugee camps, diligent outreach activities by programme staff contributed to nearly all girl and caregiver participants attending at least $75 \%$ of programme sessions. Additionally, future programme in these settings might consider adopting COMPASS' strategic approach to programme scheduling in order to maximise participation. For example, in the study setting, school sessions are divided, with approximately half of girls attending school in the morning and half attending in the afternoon; COMPASS staff worked with women and girls to ensure programme sessions and safe spaces were accessible at convenient times.

This study has a few limitations worth noting. First, all outcomes are self-reported and thus subject to disclosure bias. For example, we observe inconsistencies in reports of ever having had consensual or non-consensual sex between baseline and endline. However, these inconsistencies were not associated with treatment status and thus we do not believe they biased estimates of programme impacts. Contextual insights provided by COMPASS programme staff suggest presence of maturity bias, as willingness to share intimate details decreases as girls age in this setting.
While we acknowledge potential bias in reporting on the study's outcomes, we believe utilisation of ACASI, which has been shown to increase the disclosure of responses to questions on sexual behaviours and violence, helped to minimise such biases. ${ }^{4142}$ Second, as mentioned above, realities in the field associated with implementing an RCT in a humanitarian setting led to programme delays, resulting in overlap between the reference period for the primary outcome and the period of intervention delivery. A longer follow-up period may more clearly illuminate any changes in violence reduction. Third, although we measure severity of sexual violence by assessing various types of violence from sexual touching to forced sex, a measure of frequency would have been useful in evaluating the programme's impact in line with the theory of change. Finally, while groups were randomised in separate geographic zones of three camps, we cannot completely rule out potential spillover or contamination between treatment and control girls. Such contamination may partially explain the overall decline in self-reported past-year sexual violence in the full sample, and, if contamination did produce these reductions, it may have masked the overall effectiveness of the intervention. Despite these limitations, this study demonstrates that programming and rigorous research can successfully be conducted with adolescent girls in a refugee setting, particularly given the limited attrition rate, and contributes to the limited evidence base for adolescent girls in humanitarian settings.

\section{CONCLUSION}

To our knowledge, this is the first study to assess the impact of a life skills and safe spaces intervention for adolescent girls in a refugee setting, where physical, sexual, and emotional violence, transactional sex and child marriage are salient threats to well-being. Experiencing these events during adolescence disrupts physical and mental development and contributes to long-term health problems, and intervention at this stage in the life course remains critical. ${ }^{811}$ Findings suggest life skills programming can influence secondary outcomes that promote healthy transitions to adulthood, while further research and programmatic adaptations may be needed to fully realise changes in risk of violence.

\section{Author affiliations}

${ }^{1}$ Department of Population and Family Health, Columbia University Mailman School of Public Health, New York City, New York, USA

${ }^{2}$ Brown School at Washington University in St. Louis, St. Louis, M0, United States ${ }^{3}$ New York University Rory Meyers College of Nursing, New York City, New York, USA ${ }^{4}$ Independent Consultant, Addis Ababa, Ethiopia

${ }^{5}$ International Rescue Committee, New York City, New York, USA

Acknowledgements The authors would like to thank the adolescent girls who participated in the study, for their willingness to share their time and experiences with us.

Contributors LS is principal investigator and led manuscript development. KA led data analysis, supported manuscript development and data collection. IS supported data analysis, interpretation and manuscript development. Coinvestigators KF and LW supported conceptual development and study implementation. TTG managed oversight of data collection. AN and LW are responsible for assisting in tool 
adaptation and study implementation. All authors reviewed and approved the final manuscript prior to submission.

Funding The study was funded by the UK Department for International Development (grant \#40080602). The funders played no role in the study design; in the collection, analysis and interpretation of data; in the writing of the report; and in the decision to submit the article for publication. All authors had full access to all of the data (including statistical reports and tables) in the study and can take responsibility for the integrity of the data and the accuracy of the data analysis. The sponsor of the study had no role in study design, data collection, data analysis, data interpretation, or writing of the report. The corresponding author had full access to all the data in the study and had final responsibility for the decision to submit for publication.

Competing interests None declared.

Patient consent Not required.

Ethics approval Columbia University Medical Center IRB; Administration for Refugee and Returnee Affairs in Ethiopia; International Rescue Committee Internal Review Board.

Provenance and peer review Not commissioned; externally peer reviewed.

Data sharing statement Due to legal restrictions, data cannot be made publicly available. Data are owned by the International Rescue Committee (IRC). For more information about IRC data, please visit the following URL: . For data related inquiries please contact Kathryn Falb (Kathryn.Falb@rescue.org).

Open access This is an open access article distributed in accordance with the Creative Commons Attribution Non Commercial (CC BY-NC 4.0) license, which permits others to distribute, remix, adapt, build upon this work non-commercially, and license their derivative works on different terms, provided the original work is properly cited, appropriate credit is given, any changes made indicated, and the use is non-commercial. See: http://creativecommons.org/licenses/by-nc/4.0

\section{REFERENCES}

1. Patton GC, Sawyer SM, Ross DA, et al. From advocacy to action in global adolescent health. J Adolesc Health 2016;59:375-7.

2. UNICEF. A statistical snapshot of violence against adolescent girls. New York: United Nations Children's Fund, 2014.

3. World Health Organization,Department of Reproductive Health and Research LSoHaTM,South African Medical Research Council. Global and regional estimates of violence against women: $p$ revalence and health effects of intimate partner violence and non-partner sexual violence. Italy: World Health Organization, 2013.

4. Watts C, Zimmerman C. Violence against women: global scope and magnitude. Lancet 2002;359:1232-7.

5. Guedes A, Bott S, Garcia-Moreno C, et al. Bridging the gaps: a global review of intersections of violence against women and violence against children. Glob Health Action 2016;9:31516.

6. Stark $L$, Wessells M. Sexual violence as a weapon of war. JAMA 2012;308:677-8.

7. Catani C, Jacob N, Schauer E, et al. Family violence, war, and natural disasters: a study of the effect of extreme stress on children's mental health in Sri Lanka. BMC Psychiatry 2008;8:33.

8. Macmillan R. Violence and the life course: The consequences of victimization for personal and social development. Annu Rev Sociol $2001 ; 27: 1-22$

9. Thornberry TP, Ireland TO, Smith CA. The importance of timing: the varying impact of childhood and adolescent maltreatment on multiple problem outcomes. Dev Psychopathol 2001;13:957-79.

10. Johnson MK, Crosnoe R, Elder GH. Insights on adolescence from a life course perspective. $J$ Res Adolesc 2011;21:273-80.

11. Olofsson N, Lindqvist $\mathrm{K}$, Shaw BA, et al. Long-term health consequences of violence exposure in adolescence: a 26-year prospective study. BMC Public Health 2012;12:411

12. Springer KW, Sheridan J, Kuo D, et al. Long-term physical and mental health consequences of childhood physical abuse: results from a large population-based sample of men and women. Child Abuse Negl 2007;31:517-30.

13. Decker MR, Silverman JG, Raj A. Dating violence and sexually transmitted disease/HIV testing and diagnosis among adolescent females. Pediatrics 2005;116:e272-e6.

14. Hughes K, Lowey H, Quigg Z, et al. Relationships between adverse childhood experiences and adult mental well-being: results from an english national household survey. BMC Public Health 2016;16:222.
15. Berenson AB, Wiemann CM, McCombs S. Exposure to violence and associated health-risk behaviors among adolescent girls. Arch Pediatr Adolesc Med 2001;155:1238-42.

16. Petroni S, Patel V, Patton G. Why is suicide the leading killer of older adolescent girls? The Lancet;386:2031-2.

17. Mokdad AH, Forouzanfar MH, Daoud F, et al. Global burden of diseases, injuries, and risk factors for young people's health during 1990-2013: a systematic analysis for the Global Burden of Disease Study 2013. Lancet 2016;387:2383-401.

18. Sarnquist C, Omondi B, Sinclair J, et al. Rape prevention through empowerment of adolescent girls. Pediatrics 2014;133:e1226-32.

19. Brady M, Assaad R, Ibrahim B, et al. Providing new opportunities to adolescent girls in socially conservative settings: The Ishraq program in rural Upper Egypt. New York, NY: The Population Council, 2007.

20. Fulu E, Kerr-Wilson A, Lang J. What works to prevent violence against women and girls. Evidence review of interventions to prevent violence against women and girls. Pretoria: Medical Research Council, 2014.

21. Erulkar A, Tamrat T. Evaluation of a reproductive health program to support married adolescent girls in rural ethiopia. Afr $\mathrm{J}$ Reprod Health 2014;18:68.

22. Noble E, Ward L, French S, et al. State of the evidence: a systematic review of approaches to reduce gender-based violence and support the empowerment of adolescent girls in humanitarian settings. Trauma Violence Abuse, . 2017;1524838017699601:1524838017699 601. Violence, \& Abuse.

23. Fellmeth GL, Heffernan C, Nurse J, et al. Educational and skillsbased interventions for preventing relationship and dating violence in adolescents and young adults. Cochrane Database Syst Rev 2013;6:CD004534.

24. Asghar K, Mayevskaya $\mathrm{Y}$, Sommer M, et al. Promoting adolescent girls' well-being in pakistan: a mixed-methods study of change over time, feasibility, and acceptability, of the compass program. Prev Sci 2018:1-13.

25. Tempany $M$. What research tells us about the mental health and psychosocial wellbeing of Sudanese refugees: a literature review. Transcult Psychiatry 2009;46:300-15.

26. Schulte J, Rizvi Z. In Search of safety and solutions: somali refugee adolescent girls at sheder and aw barre camps, ethiopia. New York: Women's Refugee Commission, 2012.

27. Ennew J. Dominique Pierre Plateau DP. How to research the physical and emotional punishment of children. Bangkok, Thailand: International Save the Children Alliance, 2004.

28. Save the Children. Practice standards in children's participation. London, UK: International Save the Children Alliance, 2005.

29. Schenk K, Williamson J. Ethical approaches to gathering information from children and adolescents in international settings: guidelines and resources. Washington, DC: Population Council, 2005.

30. Falb KL, Tanner S, Ward L, et al. Creating opportunities through mentorship, parental involvement, and safe spaces (COMPASS) program: multi-country study protocol to protect girls from violence in humanitarian settings. BMC Public Health 2016;16:231.

31. Falb K, Tanner S, Asghar K, et al. Implementation of audiocomputer assisted self-interview (acasi) among adolescent girls in humanitarian settings: feasibility, acceptability, and lessons learned. Confl Health 2016;10:32.

32. Sommer M, Munoz-Laboy M, Wilkinson Salamea E, et al. How narratives of fear shape girls' participation in community life in two conflict-affected populations. Violence Against Women 2018;24:565-85.

33. Sommer M, Muñoz-Laboy M, Williams A, et al. How gender norms are reinforced through violence against adolescent girls in two conflict-affected populations. Child Abuse Negl 2018;79:154-63.

34. Runyan DK, Dunne MP, Zolotor AJ, et al. The development and piloting of the ispcan child abuse screening tool-parent version (ICAST-P). Child Abuse Negl 2009;33:826-32.

35. Abramsky T, Watts $\mathrm{CH}$, Garcia-Moreno $\mathrm{C}$, et al. What factors are associated with recent intimate partner violence? findings from the WHO multi-country study on women's health and domestic violence. BMC Public Health 2011;11:109.

36. Stark L, Asghar K, Yu G, et al. Prevalence and associated risk factors of violence against conflict-affected female adolescents: a multi-country, cross-sectional study. J Glob Health 2017;7:010416.

37. Wagman JA, Gray RH, Campbell JC, et al. Effectiveness of an integrated intimate partner violence and HIV prevention intervention in Rakai, Uganda: analysis of an intervention in an existing cluster randomised cohort. Lancet Glob Health 2015;3:e23-e33.

38. Ashburn K, Kerner B, Ojamuge D, et al. Evaluation of the responsible, engaged, and loving (real) fathers initiative on physical child punishment and intimate partner violence in northern uganda. Prevention Science 2016:1-11. 
39. Abramsky T, Devries KM, Michau L, et al. Ecological pathways to prevention: How does the SASA! community mobilisation model work to prevent physical intimate partner violence against women? BMC Public Health 2016;16:339.

40. Stark L, Sommer M, Davis K, et al. Disclosure bias for group versus individual reporting of violence amongst conflictaffected adolescent girls in DRC and Ethiopia. PLoS One 2017;12:e0174741.
41. Mensch BS, Hewett PC, Gregory R, et al. Sexual behavior and STI/HIV status among adolescents in rural Malawi: an evaluation of the effect of interview mode on reporting. Stud Fam Plann 2008;39:321-34.

42. van der Elst EM, Okuku HS, Nakamya $P$, et al. Is audio computerassisted self-interview (ACASI) useful in risk behaviour assessment of female and male sex workers, Mombasa, Kenya? PLoS One 2009;4:e5340. 